\title{
Relationships between neuroradiological and clinical features in apparently idiopathic hypopituitarism
}

\author{
Teresa Arrigo, Filippo De Luca, Mohamad Maghnie ${ }^{1}$, Alfredo Blandino, Fortunato Lombardo, Maria F Messina, \\ Malgorzata Wasniewska, Lucia Ghizzoni ${ }^{2}$ and Mauro Bozzola ${ }^{1}$ \\ Institute of Pediatrics, University of Messina, Italy, ${ }^{1}$ Institute of Pediatrics, University of Pavia, Italy, and ${ }^{2}$ Institute of Pediatrics, \\ University of Parma, Italy \\ (Correspondence should be addressed to F De Luca, Istituto di Clinica Pediatrica, Policlinico Universitario, 98100 Messina, Italy)
}

\begin{abstract}
In this study, perinatal history, postnatal auxological and clinical evolution and endocrine features were retrospectively evaluated in 49 children, adolescents and young adults with apparently idiopathic hypopituitarism. They were divided into two groups according to magnetic resonance images: 32 patients with isolated pituitary hypoplasia (group A) and 17 with pituitary stalk interruption syndrome (group B). The aim of the study was to assess whether these neuroradiological pictures are associated with specific endocrine and clinical patterns. No significant difference in terms of gestational age, intrauterine growth and rates of adverse perinatal events was found between the two groups. Clinical signs documenting the existence of pituitary dysfunction in utero or shortly after birth were either slightly (micropenis, cryptorchidism, cholestatic jaundice) or significantly (hypoglycemia) more frequent in patients in group B. Although diagnosis of hypopituitarism was made significantly earlier in patients in group B, height deficiency at diagnosis was similar in both groups. Endocrine investigations revealed a more severe and widespread impairment of pituitary function among those in group B. The main conclusion is that the postnatal clinical course is more severe when growth hormone deficiency is associated with pituitary stalk interruption syndrome than when the pituitary is only reduced in height, probably because of the more severe and widespread impairment of pituitary function in the former cases.
\end{abstract}

European Journal of Endocrinology 139 84-88

\section{Introduction}

Magnetic resonance imaging (MRI) is a non-invasive technique that facilitates good visualization of both the hypophysis and the pituitary stalk (1-4), thus improving our understanding of the pathophysiology of apparently idiopathic growth hormone deficiency (GHD) (5-8).

The most frequent defects observed by MRI in children with non-tumoral GHD are isolated pituitary hypoplasia and pituitary stalk interruption syndrome i.e. pituitary stalk interruption associated with anterior pituitary hypoplasia or no visible anterior lobe.

Pituitary stalk interruption syndrome seems to be more strongly associated with perinatal adverse events (9-10) and has been reported more frequently in patients with multiple pituitary hormone deficiency than in those with isolated GHD $(6,8,9-12)$. Nevertheless, the existence of significant relationships between neuroradiological findings and either perinatal events or endocrine function in hypopituitarism has not been confirmed by others and the subject remains controversial $(12-16)$.

In order to shed further light on the pathophysiology of apparently idiopathic GHD, we retrospectively evaluated perinatal history, postnatal clinical evolution and endocrine features in two groups of patients with non-tumoral and apparently idiopathic hypopituitarism and two different neuroradiological patterns, either isolated pituitary hypoplasia or pituitary stalk interruption syndrome.

\section{Patients and methods}

Our retrospective study involved 49 patients (35 males) with non-tumoral and apparently idiopathic GHD who were seen in our three clinics over the past 10 years and were diagnosed at a mean age of $5.6 \pm 5.2$ years (range $0.1-25.0$ years). All the patients admitted to the study fulfilled the following inclusion criteria: height $<-2$ SDS (standard deviation score) (mean $-3.6 \pm 1.3$, range -2.1 to $-7.1 \mathrm{SDS})$; growth hormone $(\mathrm{GH})$ peak in response to at least two pharmacological stimulation tests (clonidine, insulin, arginine, L-dopa) $<10.0 \mu \mathrm{g} / \mathrm{l}$ (mean 3.0 \pm 2.6 , range $0.1-8.9 \mu \mathrm{g} / \mathrm{l}$ ); presence of neuroradiological abnormalities such as isolated pituitary hypoplasia or pituitary stalk interruption, as demonstrated by MRI.

A tumoral cause of GHD was excluded by both clinical and neuroradiological evaluation. Many causes of 
hereditary GHD were excluded on the basis of the following: absence of family history of hypopituitarism; non-consanguineity of patients' parents; absence of known immunodeficiency or history of recurrent infections in GHD males; absence of GH antibodies in response to $\mathrm{GH}$ treatment; normal prolactin secretion in patients with both GH and thyrotropin (TSH) deficiency.

The patients were classified into two groups according to the MR images: group A, 32 patients (24 males) with isolated pituitary hypoplasia; group B, 17 patients (11 males) with pituitary stalk interruption, associated with pituitary hypoplasia in 12 or with no visible anterior pituitary lobe in the remaining five.

Patients' perinatal history and their clinical, auxological and endocrine data were obtained from hospital records. Breech delivery and emergency cesarean section were considered to be abnormal deliveries. An Apgar score $\leq 7$ at $5 \mathrm{~min}$ after birth was considered to be an indication of neonatal asphyxia. Abnormal deliveries and neonatal asphyxia were considered adverse perinatal events. The auxological data at birth, according to gestational age, were evaluated with respect to the standards of Usher \& McLean (18), whereas the standards of Tanner \& Whitehouse (19) were used to evaluate height at the time of diagnosis of the pituitary deficiency.

$\mathrm{GH}$ assays were performed in all cases using the same commercial kits based on polyclonal antibodies (Biodata, Milan, Italy). Thyroid and adrenal functions were evaluated in all patients, leading to a classification of isolated GHD or multiple pituitary hormone deficiency. The pituitary-thyroid axis function was investigated by evaluating serum thyroxine $\left(\mathrm{T}_{4}\right)$, free $\mathrm{T}_{4}$, tri-iodothyronine $\left(\mathrm{T}_{3}\right)$, free $\mathrm{T}_{3}$ and TSH baseline serum concentrations and by a TSH-releasing-hormone (TRH) test $\left(200 \mu \mathrm{g} / \mathrm{m}^{2}\right.$ i.v.): reduced free $\mathrm{T}_{4}$ values $(<12 \mathrm{pmol} / \mathrm{l})$ with a delayed TSH response after injection of TRH (TSH value at $60 \mathrm{~min}$ greater than that obtained at 20-30 min) was considered to indicate hypothalamic hypothyroidism.

Adrenal function was estimated as basal serum cortisol (normal values $265-365 \mathrm{nmol} / \mathrm{l}$ ) and corticotropin (normal values $2.2-17.6 \mathrm{pmol} / \mathrm{l}$ ) concentrations at $0800 \mathrm{~h}$, and as the serum cortisol response to insulininduced hypoglycemia (normal response was defined as an increase above baseline cortisol concentrations of more than $190 \mathrm{nmol} / \mathrm{l}$ or an increase to a peak value greater than $550 \mathrm{nmol} / \mathrm{l})$. Diagnosis of gonadotropin deficiency was made on clinical grounds only in newborns with both microphallus and cryptorchidism; in other cases it was suggested by the absence of physical signs of pubertal development at advanced bone age ( $>12$ years in girls and $>13.5$ years in boys) and confirmed by biochemical data (prepubertal baseline serum gonadotropin concentrations and no increase after injection of gonadotropin-releasing hormone $100 \mu \mathrm{g} / \mathrm{m}^{2}$ i.v. $)$.

The MRI scan was routinely performed in all the patients included in our series before hormonal treat- ment was begun, in order to identify the cause of their hypopituitarism. At the time of the scan, no patient in the entire series, including the one aged 25 years, had entered puberty. As the size of the pituitary may physiologically increase at the time of puberty (15), patients in group A underwent another MRI scan after completing their spontaneous or induced puberty. The second scan was performed in order to re-evaluate diagnosis of isolated pituitary hypoplasia, and was not performed in patients in group B, as they had exhibited, not isolated pituitary hypoplasia, but multiple morphological abnormalities.

MRI studies were performed with a 0.5-T superconducting unit. A conventional spin-echo pulse sequence was adopted using a standard pituitary protocol. Sagittal and coronal T1-weighted images (repetition time $310 \mathrm{~ms}$, echo time $15 \mathrm{~ms}$, four excitations) were obtained by the interleaving method using a head coil with a $24-\mathrm{cm}$ field of view, a $256 \times 256$ pixel acquisition matrix, and $3-\mathrm{mm}$ section thickness. The images were evaluated specifically for adenohypophysis size and morphology, morphological characteristics of the pituitary stalk and presence of neurohypophysis in the posterior part of the sellar fossa. The height of the pituitary gland was determined on the midsagittal T1-weighted image by measuring the greatest distance between the base and the top of the gland using a cursor on the display. Pituitary height measurements were compared with published normal values for age as obtained using the same imaging and laboratory studies. A pituitary was considered to be hypoplastic when the gland height was less than -2 s.D. for age (20).

The MR images were evaluated independently by three experienced neuroradiologists from our three Universities, who agreed as to the criteria for evaluating the parameters studied.

Comparisons between groups were performed by Student's unpaired t-test, by Pearson's $\chi^{2}$ test and, when appropriate, by Mann-Whitney test. $P$ values $<0.05$ were considered significant. Data are expressed as means \pm S.D.

\section{Results}

In fulfilment of one of the inclusion criteria, all the patients enrolled for this study exhibited a subnormal pituitary height at the time of their first MRI scan (mean $-3.3 \pm 0.9$ SDS; range -2.1 to -5.8$)$, with no significant difference between groups A (mean -3.3 \pm 0.8 SDS; range -2.4 to -5.8 ) and $B$ (mean $-3.2 \pm 1.0$; range -2.1 to -5.1 ). In patients in group $A$, pituitary height remained subnormal at the second scan (mean $-2.8 \pm 0.9$ SDS; range -2.1 to -5.2 ).

Perinatal history and endocrine features of the patients in both groups, and their auxological and clinical data are summarized in Table 1.

Prematurity was recorded in four patients (two in each group). In the entire series, adverse perinatal 
Table 1 Perinatal, endocrine, auxological and clinical data of patients.

\begin{tabular}{lcccc}
\hline & $\begin{array}{c}\text { Entire series } \\
(n=49)\end{array}$ & $\begin{array}{c}\text { Group A } \\
(n=32)\end{array}$ & $\begin{array}{c}\text { Group B } \\
(n=17)\end{array}$ & $\boldsymbol{P}$ \\
\hline Gestational age (weeks) & $39.5 \pm 1.6$ & $39.6 \pm 1.4$ & $39.2 \pm 2.0$ & $\mathrm{NS}$ \\
Abnormal deliveries (\%) & 34.7 & 34.3 & 35.3 & $\mathrm{NS}$ \\
Neonatal asphyxia (\%) & 26.5 & 28.1 & 23.5 & $\mathrm{NS}$ \\
GH peaks ( $\mu$ g/l) & $3.0 \pm 2.6$ & $3.8 \pm 2.7$ & $1.6 \pm 1.7$ & $<0.0005$ \\
MPHD (\%) & 36.7 & 21.9 & 64.7 & $<0.005$ \\
Birth length (SDS) & $-1.3 \pm 0.9$ & $-1.3 \pm 0.9$ & $-1.4 \pm 1.0$ & $\mathrm{NS}$ \\
Height at diagnosis (SDS) & $-3.6 \pm 1.2$ & $-3.4 \pm 1.1$ & $-4.0 \pm 1.5$ & $\mathrm{NS}$ \\
Age at diagnosis (years) & $5.6 \pm 5.2$ & $6.9 \pm 5.9$ & $3.2 \pm 2.3$ & $<0.0025$ \\
Microphallus (\%) & 20.0 & 12.5 & 36.4 & $\mathrm{NS}$ \\
Cryptorchidism (\%) & 14.3 & 8.3 & 27.3 & $\mathrm{NS}$ \\
Hypoglycemia (\%) & 14.3 & 3.1 & 35.3 & $<0.005$ \\
Cholestatic jaundice (\%) & 6.1 & 3.1 & 11.8 & $\mathrm{NS}$ \\
\hline
\end{tabular}

MPHD, multiple pituitary hormone deficiency; NS, not significant.

events were clearly more frequent than in the general population: in our three Hospitals, the average frequencies of abnormal deliveries (as previously defined) and neonatal asphyxia over the past 10 years were approximately $15 \%$ and 3\% respectively. No significant difference in terms of gestational age and rates of adverse perinatal events was found between the two groups (Table 1).

Endocrine investigations revealed a more severe and widespread impairment of pituitary function in patients in group B, who exhibited both lower $\mathrm{GH}$ peaks after pharmacological stimulations and more frequent involvement of other pituitary hormones (Table 1). GH responses were very severely blunted (peak $<3.0 \mu \mathrm{g} / \mathrm{l}$ ) in many patients in both the groups (14 of 17 with pituitary stalk interruption syndrome, compared with 13 of 32 without the syndrome; $\chi^{2}=7.8, P<0.01$ ).

Both the average birth length and the prevalence of subnormal (<-2 SDS) birth length (6 of 17 compared with 6 of 32) were similar in the two groups, as were the patients' heights at the time of diagnosis of their condition (Table 1). Diagnosis of GHD, however, was made significantly earlier in patients in group B (mean age at diagnosis $3.2 \pm 2.3$ years; range $0.1-10.0$ years) than in those in group A (mean $6.9 \pm 5.9$; range $0.1-$ 25.0 years; Table 1 ).

Clinical symptoms of the existence of pituitary dysfunction in utero or shortly after birth were observed in patients in both groups, although they were either slightly (genitalia abnormalities, cholestatic jaundice) or significantly (hypoglycemia) more frequent in those group B (Table 1).

Midline developmental defects were found in only two patients (one with cleft palate in group A and one with septo-optic dysplasia in group B).

\section{Discussion}

MRI is an additional and useful tool in the evaluation of patients with GHD, in whom a diagnosis of permanent hypopituitarism might be in doubt if both the anatomy and the height of the pituitary are normal $(5,21)$.
Patients were admitted to this study only if MRI revealed anterior pituitary hypoplasia. All were subsequently divided into two groups according to the presence or absence of pituitary stalk interruption. This study design enabled us to evaluate: a) the specific influences of pituitary stalk interruption syndrome on prenatal, perinatal and postnatal history of GHD patients; b) the relationships between pituitary stalk interruption syndrome and pituitary function; and c) whether GHD syndrome is characterized by different and peculiar endocrine and clinical patterns in the patients with either isolated pituitary hypoplasia or pituitary stalk interruption syndrome.

Pituitary failure began during intrauterine life in many infants with either isolated pituitary hypoplasia or pituitary stalk interruption syndrome, as suggested by the following perinatal data recorded in the patients of both groups: 1) the greater than normal frequency of infants with short birth length, which confirms that GH may have a role in prenatal growth $(22,23) ; 2)$ the pathological prevalence of both microphallus and bilateral cryptorchidism in boys; 3 ) the abnormally high frequency of adverse perinatal events, which might be regarded as a complication of prenatal hypopituitarism (23-27), at least in those patients with intrauterine growth retardation or intrauterine developmental defects.

Neither prenatal nor perinatal events were significantly affected by pituitary stalk interruption syndrome in patients with GHD - an observation that is in contrast with the findings of other studies $(8,24)$, but is strongly supported by the following: 1) pregnancy duration and rates of adverse perinatal events were similar in our patients both with or without pituitary stalk interruption syndrome; 2) length deficiency at birth was very similar in the two groups; 3) developmental defects of the genitalia were apparently more frequent in patients in group B, but this difference was not statistically significant; 4) midline malformations were very rare in both groups.

In contrast, the postnatal clinical course was significantly more severe in our patients with pituitary 
stalk interruption syndrome than in those with isolated pituitary hypoplasia, as has also been reported by others $(5,14,28)$. GHD children with pituitary stalk interruption syndrome more frequently experienced hypoglycemic attacks, a complication observed in only a few who did not have the syndrome. In addition, cholestasis occurred slightly more frequently in patients with pituitary stalk interruption syndrome. Probably as a consequence of the more frequent postnatal clinical complications, diagnosis of hypopituitarism was made earlier in the patients with pituitary stalk interruption syndrome than in those in group A. In spite of the differences in age at diagnosis, height deficiency at the time of diagnosis was very similar in the two groups of subjects, which strongly suggests that growth velocity during the first years of life had been significantly lower in the children with pituitary stalk interruption syndrome than in those with isolated pituitary hypoplasia.

In our series, as in previous reports $(1,2,5,6,8,10$, $12,13,15)$, pituitary function was more severely impaired in the patients with pituitary stalk interruption syndrome, as revealed by both the blunted $\mathrm{GH}$ responses to provocative tests and the frequent involvement of other pituitary hormones. In contrast, in the patients with isolated pituitary hypoplasia, GH secretion was less severely impaired and GHD was associated with other pituitary hormone deficiencies in only a few of them.

The major conclusion that can be drawn from the present study is that the postnatal clinical course is more severe when GHD is associated with pituitary stalk interruption syndrome than when the pituitary is only reduced in height, probably because of the more severe and widespread impairment of pituitary function in patients with pituitary stalk interruption syndrome.

Other significant inferences suggested by the analysis of our results are that, in GHD patients with subnormal pituitary height, hypopituitarism may start in utero, irrespective of whether pituitary hypoplasia is isolated or associated with other MRI abnormalities; that perinatal history is frequently similar in GHD patients with either isolated pituitary hypoplasia or pituitary stalk interruption syndrome; and that both isolated pituitary hypoplasia and pituitary stalk interruption syndrome may have a prenatal etiology in some patients.

\section{References}

1 Colombo N, Berry I, Kucharczyk J, Kuch Arczyk W, De Grood J, Karson $\mathrm{T}$ et al. Posterior pituitary gland: appearance on MR images in normal and pathologic states. Radiology 1987165 481-485.

2 Di Natale B, Scotti G, Pellini C, del Maschio A, Triulzi F, Petecca C et al. Empty sella in children with pituitary dwarfism: does it exist? Pediatrician 198714 246-252.

3 Zucchini S, di Natale B, Ambrosetto P, de Angelis R, Cacciari E \& Chiumello G. Role of magnetic resonance imaging in hypothalamic-pituitary disorders. Hormone Research 199544 (suppl 3) 8-14
4 Weiner SN, Rzezotarski MS, Droege RT \& Pearlstein AE. Measurement of pituitary gland height with MR imaging. American Journal of Neuroradiology 19856 717-722.

5 Argyropoulou M, Perignon F, Brauner R \& Brunelle F. Magnetic resonance imaging in the diagnosis of growth hormone deficiency. Journal of Pediatrics 1992120 886-891.

6 Bressani N, di Natale B, Pellini C, Triulzi F, Scotti G \& Chiumello G. Evidence of morphological abnormalities of growth hormone deficient children: a combined magnetic resonance imaging and endocrine study. Hormone Research 199034 189-192.

7 Pellini C, di Natale B, de Angelis R, Bressani N, Scotti G, Triulzi Fet al. Growth hormone deficiency in children: role of magnetic resonance imaging in assessing aethiopathogenesis and prognosis in idiopathic hypopituitarism. European Journal of Pediatrics 1990149 536-541.

8 Maghnie M, Triulzi F, Larizza D, Preti P, Priora C, Scotti G et al. Hypothalamic-pituitary dysfunction in growth-hormonedeficient patients with pituitary abnormalities. Journal of Clinical Endocrinology and Metabolism 1991 73 79-83.

9 Triulzi F, Livian S \& di Natale B. Pituitary and brain abnormalities in pituitary dwarfs: MR imaging findings in 49 patients. 74 th Annual Meeting of the RSNA, Chicago, 1988. Abstract 494.

10 Kikuchi K, Fujisawa I, Momoi T, Yamanaka C, Kaji M, Nakano Y et al. Hypothalamic-pituitary function in growth hormonedeficient patients with pituitary stalk transection. Journal of Clinical Endocrinology and Metabolism 198867 817-823.

11 Maghnie M, Triulzi F \& Larizza D. Hypothalamic-pituitary dwarfism: comparison between MR imaging and CT findings. Pediatric Radiology $199020229-235$.

12 Scotti G, Triulzi F, Chiumello G \& di Natale B. New imaging technique in endocrinology: magnetic resonance of the pituitary gland and sella turcica. Acta Paediatrica Scandinavia 1989356 (suppl) 5-14.

13 Fujisawa I, Kikuchi K, Nishimura K, Togaschi K, Itoh K, Noma S et al. Transection of the pituitary stalk: development of an ectopic posterior lobe assessed by MR imaging. Radiology $1987165487-$ 489.

14 Marwaha R, Menon PSN, Jena A, Pant C, Sethi AK \& Gapra ML. Hypothalamo-pituitary axis in isolated growth hormonedeficient patients born by normal delivery. Journal of Clinical Endocrinology and Metabolism 199274 654-659.

15 Bozzola M, Adamsbaum C, Biscaldi I, Zecca M, Cisternino M, Genovese $\mathrm{E}$ et al. Role of magnetic resonance imaging in the diagnosis and prognosis of growth hormone deficiency. Clinical Endocrinology $19964521-26$.

16 Smet MH, de Zegher F, Vanderschueren-Lodeweyck M \& Marchal G. Infantile hypopituitarism: etiological variability evidenced by MRI. European Radiology 19922 57-61.

17 Abrahams JJ, Trefelner E \& Boulware SD. Idiopathic growth hormone deficiency: MR findings in 35 patients. American Journal of Neuroradiology 199112 155-160.

18 Usher R \& McLean F. Intrauterine growth of live-born Caucasian infants at sea level: standards obtained from measurement in seven dimensions of infant born between 25 and 44 weeks of gestation. Journal of Pediatrics 196974 901-910.

19 Tanner JM \& Whitehouse RH. Clinical longitudinal standards for height, weight, height velocity, weight velocity and the stages of puberty. Archives of Diseases in Childhood 197651 170-179.

20 Argyropoulou M, Perignon F, Brunelle F, Brauner R \& Rappaport R. Height of normal pituitary gland as a function of age evaluated by magnetic resonance. Pediatric Radiology 199122 47-49.

21 Michalacos S, Hadjiathanasiou C, Theodoridis C, Athanasopoulou A, Papatheodourou A, Karris C et al. The value of magnetic resonance imaging in the diagnosis of idiopathic growth hormone deficiency (Abstract). Hormone Research 199646 (suppl 2) 135.

22 Gluckman PD, Gunn AJ, Wray A, Cutfield WS, Chatelein PG, Guilbaud $\mathrm{O}$ et al. Congenital idiopathic growth hormone deficiency associated with prenatal and early postnatal growth failure. Journal of Pediatrics 1992121 920-923.

23 De Luca F, Bernasconi S, Blandino A, Cavallo L \& Cisternino M. Auxological, clinical and neuroradiological findings in infants 
with early onset growth hormone deficiency. Acta Paediatrica $199584561-565$.

24 Triulzi F, Scotti G, di Natale B, Pellini C, Lukezic M \& Scognamiglio M. Evidence of a congenital midline brain anomaly in pituitary dwarfs: a magnetic resonance imaging study in 101 patients. Pediatrics 199493 409-416.

25 Van Hauthem H, Toppet V \& Van Vliet G. Congenital hypopituitarism: results of pituitary stimulation tests and of magnetic resonance imaging in a newborn girl. European Journal of Pediatrics 1992151 174-176.

26 Brown RS, Bathia V \& Hayes E. An apparent cluster of congenital hypopituitarism in central Massachussetts: magnetic resonance imaging and hormonal studies. Journal of Clinical Endocrinology and Metabolism 199172 12-18.

27 Maghnie M, Larizza D, Triulzi F, Sampaolo P, Scotti G \& Severi F. Hypopituitarism and stalk agenesis: a congenital syndrome worsened by breech delivery? Hormone Research 199135 104-108.

28 Kuroiwa T, Okaba Y, Hasno K, Yasumori K, Mizushima A \& Masuda K. MR imaging of pituitary dwarfism. American Journal of Neuroradiology $199112161-164$.

Received 17 November 1997

Accepted 16 March 1998 